\author{
Y.-F. CHAU ${ }^{1, \infty}$ \\ Y.-S. SUN ${ }^{1}$ \\ D.P. TSAI ${ }^{2}$ \\ T.-J. YANG ${ }^{3}$
}

\title{
The optical properties between an incident wave and the active layer of a bubble-pit $\mathrm{AgO}_{x}$-type super-resolution near-field structure
}

\footnotetext{
${ }^{1}$ Department of Electronic Engineering, Ching Yun University, Chien-Hsin Rd., Jung-Li, 320 Taiwan, R.O.C.

2 Department of Physics and Center for Nanostorage Research, National Taiwan University,

Taipei, Taiwan, R.O.C.

${ }^{3}$ Department of Electrophysics, National Chiao-Tung University, HsinChu 300, Taiwan, R.O.C.
}

\section{Received: 12 April 2007/Accepted: 2 May 2007 \\ Published online: 21 June 2007 • @ S Springer-Verlag 2007}

ABSTRACT The deformation and plasmon effects of collective localized surface plasmons between incident light and bubblepit $\mathrm{AgO}_{x}$-type super-RENS structure have been studied using finite-difference time-domain (FDTD) method. We find that the polarization, wavelength of incident light, and particle sizes of Ag nanoparticles are sensitive to the plasma resonance. The Ag nanoparticles inside the bubble-pit $\mathrm{AgO}_{x}$-type super-RENS structure give the additional outer boundaries to the motion of the Ag nanoparticles, and excite more evanescent field which located in the far edge of the bubble from the optical axis of the incident beam. The optical properties between active layer and incident light with polarization direction, different wavelengths, and varied particle sizes of Ag nanoparticles exhibits nonlinear optical behavior in the near field. The far-field signals of different wavelength of incident light confirm the relation between highly localized near-field distributions and enhanced resolution of far-field signals. The subwavelength recording marks smaller than the diffraction limit were distinguishable since the Ag nanoparticles with high localized fields transferred evanescent waves to detectable signals in the far field.

PACS 42.79.Vb; 71.15.Rn; 72.15.Rn; 73.22.-f; 73.22.Lp; 78.67.Bf; 73.20.Mf

\section{Introduction}

A high-density optical storage system with more than $100 \mathrm{~GB}$ capacity is required for multimedia systems such as internet broadband servers and high-definition video systems (HDTV). The super-resolution near-field structure (super-RENS) [1-5] disc is a promising candidate for the next-generation optical disc to provide high data storage capacity. Today, the novel emergent technique of near-field optical data storage, allows the writing and/or reading of information bits, which are smaller than the resolution limit of the optical head, without increasing the complexity of the detecting system. Such a technique is very attractive because of its compatibility with existing players/recorders. Near-field optical data storage techniques depend on the evanescent coupling of light to the data layer of the opti-

E-mail: yfc01@cyu.edu.tw cal storage medium to read and write data marks that are smaller than the diffraction limit of conventional optical data storage systems. In recent years, there has been increased attention given to techniques that may overcome this limit.

The discovery of optical near-field generation by plasmons excited in thin $\mathrm{AgO}_{x}$ layers has made these a key component in advanced optical applications. An $\mathrm{AgO}_{x}$ SuperRENS structure is composed of an active layer $\left(\mathrm{AgO}_{x}\right.$ thin film) that is sandwiched between two dielectric films ( $\mathrm{ZnS}$ $\mathrm{SiO}_{2}$ ), and this three-layer stack is deposited directly onto the phase-change data layer $\left(\mathrm{Ge}_{2} \mathrm{Sb}_{2} \mathrm{Te}_{5}\right)$ of a disk. One of the dielectric layers is directly adjacent to a polycarbonate layer acting as an insulating layer; the other dielectric layer is the cover layer. If a storage medium incorporates a Super-RENS structure, the light spot that probes the data layer is smaller than the size of the focused spot that is incident on the cover layer of the Super-RENS structure. The optical aperture serves as a probe on the disk like that for scanning near-field optical microscopy (SNOM). After recording on a phase-change layer, small marks are reproduced through the aperture. The particles, instead of the aperture, function as the scattering center, which is the key to inducing the super-resolution phenomenon. The $15 \mathrm{~nm} \mathrm{AgO}_{x}$ thin films are considered to be nonlinear optical layers which control the near-field optical aperture. Recently, several studies have explored this nonlinear near-field optical property; [6-9] however, the influence of the deformed Super-RENS structure does not seem to be appropriately considered. The active layer $\left(\mathrm{AgO}_{x}\right.$ film $)$ of the $\mathrm{AgO}_{x}$-type Super-RENS structure is deformed after a writing process, as observed in experimental work [10,11]. The silver $(\mathrm{Ag})$ nanoparticles spread out in the bubble pit (deformed $\mathrm{AgO}_{x}$ film) that is, in addition, coincidentally formed at the focus spot.

To examine this nonlinear near-field optical property and match it to a realistic situation, we study the relationship between the active layer (bubble pit) and incident light for various diameters of $\mathrm{Ag}$ nanoparticles in the deformed $\mathrm{AgO}_{x^{-}}$ type Super-RENS structure using the two-dimensional finitedifference time-domain (FDTD) method. In addition, the farfield signals of different wavelengths of incident light confirm the relation between highly localized near-field distributions and enhanced resolution of far-field signals. Our results indicate a close connection between the surface plasmon effects of 
Ag nanoparticles and the nonlinear near-field optical property observed in experiments.

\section{Simulation models}

Figure 1 shows bubble pit marks formed in the active layer (deformed $\mathrm{AgO}_{x}$ film) and $\mathrm{Ag}$ nanoparticles distributed randomly inside the bubble. We assume that the $\mathrm{Ag}$ particles are of the same size and randomly distributed in the deformed $\mathrm{AgO}_{x}$ layer. The distribution density of Ag nanoparticles near the top surface of the bubble pit is lower than that near the base of the bubble pit. The layer structure is, from bottom to top, polycarbonate $(n=1.5) / \mathrm{ZnS}-\mathrm{SiO}_{2}(n=2.25+\mathrm{i} 0.01) / \mathrm{AgO}_{x}(n=$ $2.270+\mathrm{i} 0.08) / \mathrm{ZnS}-\mathrm{SiO}_{2} / \mathrm{Ge}_{2} \mathrm{Sb}_{2} \mathrm{Te}_{5}$ (GST, crystal phase, $\left.n_{\mathrm{c}}=4.01+\mathrm{i} 3.61\right) / \mathrm{ZnS}-\mathrm{SiO}_{2} /$ air $(n=1)$. The figure shows that the layer structure between the insulating layer (the second $\mathrm{ZnS}-\mathrm{SiO}_{2}$ ) and the record mark (GST) is deformed after the writing process. The nanosize Ag nanoparticles inside the bubble pits are embedded in the $\mathrm{AgO}_{x}$ layer and are assumed to move under the influence of the readout laser beam, as shown by previous experimental work [12]. The dispersive behavior of Ag and GST is simulated by the Lorentz model [13]. The $\mathrm{AgO}_{x}$ layer is sandwiched between the insulating $\mathrm{ZnS}-\mathrm{SiO}_{2}$ layers and a GST-alloy phase-change data layer is located directly above this trilayer structure. The other parameters that were used were polarization (transverse magnetic (TM)-polarization, the electric field component normal to the Ag nanoparticle surface), cell size ( $\Delta x=\Delta y=$ $0.5 \mathrm{~nm})$, and the refractive index of $\mathrm{Ag}(n=0.055+\mathrm{i} 4.44)$. The incident Gaussian beams FWHM was adjusted such that $\mathrm{FWHM}=0.51 \lambda / \mathrm{NA}[14]$. The phase-change data layer is in

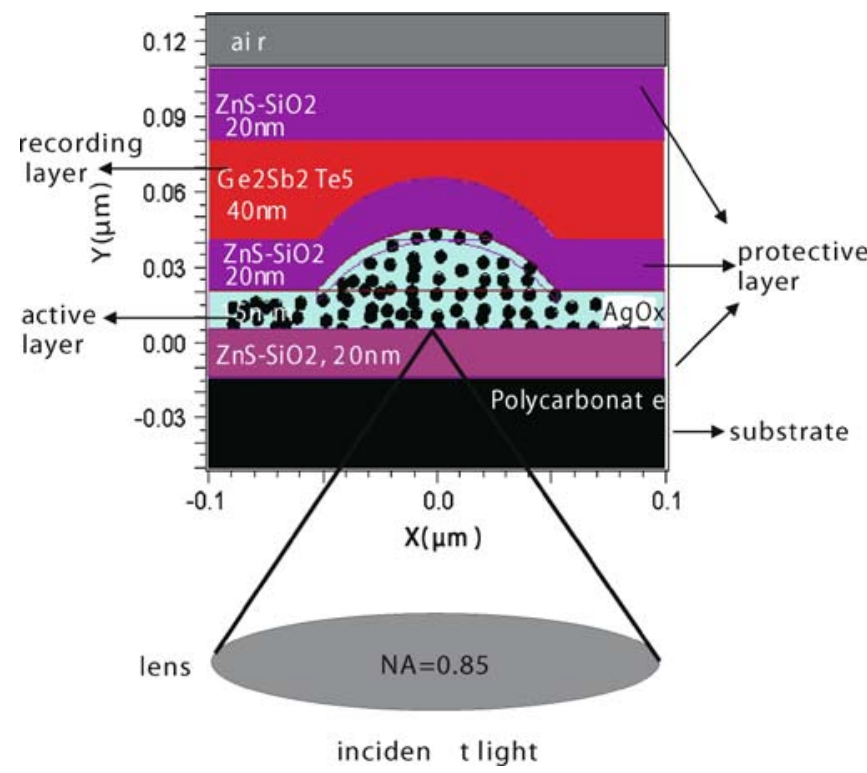

FIGURE 1 Scheme of bubble pit marks formed at the active layer (deformed $\mathrm{AgO}_{x}$ film), and $\mathrm{Ag}$ nanoparticles distributed randomly inside the bubble. The Ag particles are of the same size and randomly distributed in the deformed $\mathrm{AgO}_{x}$ layer. The distribution density of $\mathrm{Ag}$ nanoparticles near the top surface of the bubble pit is lower than that near the base of the bubble pit. The layer structure is, from bottom to top, polycarbonate $(n=1.5) / \mathrm{ZnS}$ $\mathrm{SiO}_{2}(n=2.25+\mathrm{i} 0.01) / \mathrm{AgO}_{x}(n=2.270+\mathrm{i} 0.08) / \mathrm{ZnS}-\mathrm{SiO}_{2} / \mathrm{Ge}_{2} \mathrm{Sb}_{2} \mathrm{Te}_{5}$ $\left(\mathrm{GST}\right.$, crystal phase, $\left.n_{\mathrm{c}}=4.01+\mathrm{i} 3.61\right) / \mathrm{ZnS}-\mathrm{SiO}_{2} /$ air $(n=1)$ an on-mark situation. The on-mark situation means that the incident light focuses on the recording mark, and the off-mark situation means that the incident light focuses between two marks. The numerical aperture (NA) of the objective lens used is 0.85 .

To study the far-field optical properties induced by the enhancements of localized surface plasmons, a near-to-far field transformation was used to obtain the far-field response from near-field results $[15,16]$. The method is accurate within the limits of the Kirchoff approximation. A Fourier transform is applied to the fields in the sampling plane to obtain the angular distribution of the radiation in the far-field. As described in [15], the expression of an efficient transformation to get the angular distribution of the electric fields in the far-field is given by the integral

$$
\begin{gathered}
E_{y}^{\mathrm{scat}}(r, \theta)=\frac{1}{2 \sqrt{\lambda r}} \int_{S} \mathrm{~d} y^{\prime} \exp \left[j k_{0} \sin (\theta) y^{\prime}\right] \times\left[E_{y}\left(x, y^{\prime}\right)\right. \\
\left.+\eta H_{z}\left(x, y^{\prime}\right) \cos \theta\right],
\end{gathered}
$$

where $S$ is the sampling plane, $\theta$ is the discrete angle defined by the normal to the incident plane and the observation point at the detector on a circle of radius $r$. The intensities are integrated within the numerical aperture of the objective lens. In order to correlate the simulated results with the experimental results, differences of the far-field intensity between on-mark and off-mark cases were calculated for various mark sizes. The differences of far-field intensity between on-mark and off-mark situations was calculated by $10 \times \log \left(\mid I_{\text {on }}-\right.$ $I_{\text {off }} \mid / I_{\text {no-mark }}$ ), where $I_{\text {on }}$ is the far-field intensity for the onmark case, $I_{\text {off }}$ is the far-field intensity for the off-mark case, and $I_{\text {no-mark }}$ is the far-field intensity for the case without any marks. The far-field difference signals could demonstrate the capability of super-RENS disks to distinguish subwavelength recording marks.

\section{Results and discussion}

As studied by our research group [17], the density of the Ag nanoparticles can be controlled by increasing or decreasing the incident laser power in the photodissociation process. A lower nanoparticles density shows less interaction among the nanoparticles. If the density of nanoparticles is increased, the local fields are coupled together and produce more evanescent fields from adjacent nanoparticles. Figure 2a and $\mathrm{b}$ show the near-field distributions of $\mathrm{Ag}$ nanoparticles of deformed and normal (underformed) $\mathrm{AgO}_{x}$-type Super RENS structures, respectively. The total number of nanoparticles is 82 in the deformed type and 57 in the normal type, and each nanoparticle has a diameter of $4 \mathrm{~nm}$. The wavelength of incident light is $\lambda=650 \mathrm{~nm}$ for TM polarization. In Fig. 2a and $\mathrm{b}$, highly localized enhancements and interactions are produced between adjacent $\mathrm{Ag}$ nanoparticles in the near-field zone. When the free electrons of an Ag nanoparticle oscillate collectively in resonance with the incident light, the interaction may constitute a surface plasmon polariton (SPP). The field enhancement is due to SPPs. Each embedded Ag nanoparticle behaves like a dipole and a very strong scattering center. The local fields from adjacent nanoparticles are 

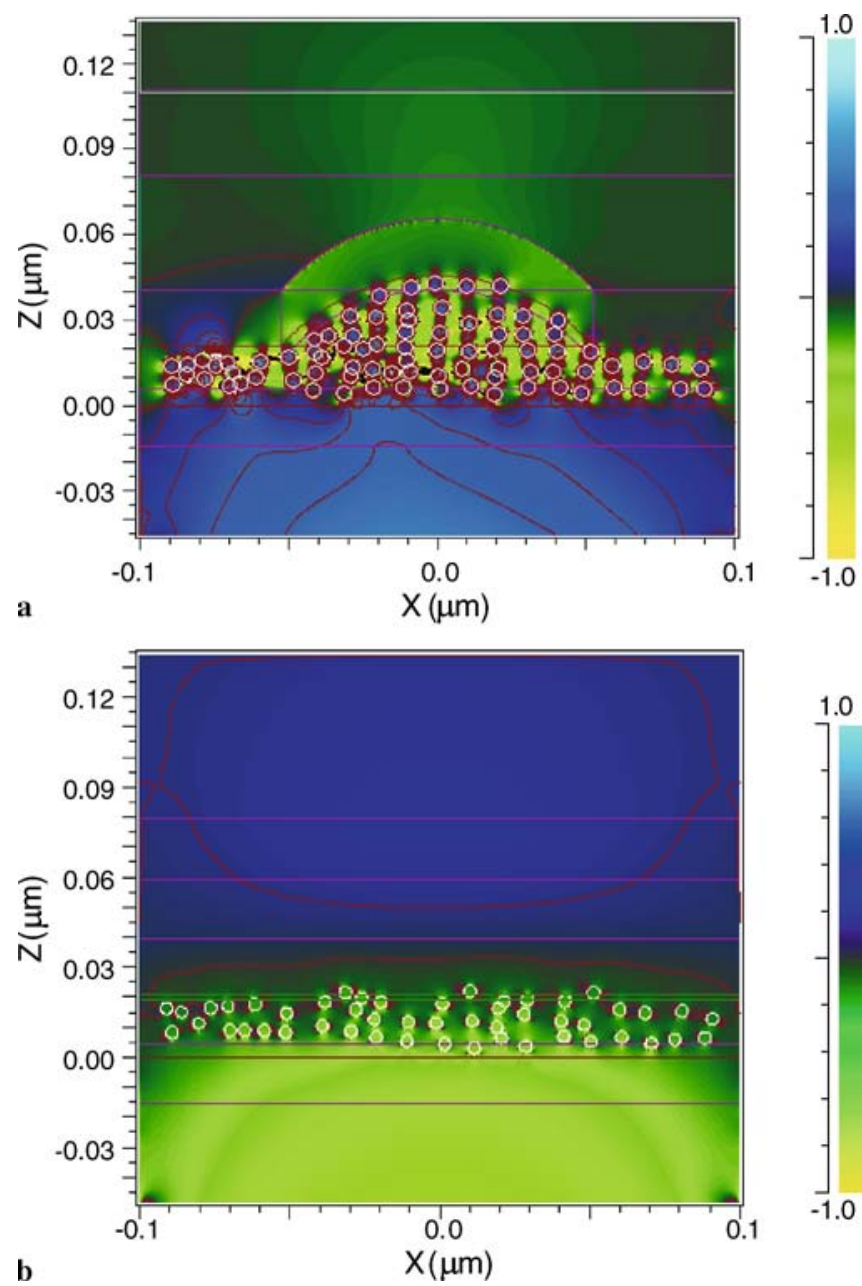

FIGURE 2 Calculated results of near-field distributions of Ag nanoparticles in deformed and normal (underformed) $\mathrm{AgO}_{x}$-type Super-RENS structure. The total number of Ag nanoparticles is 82 in the deformed type and 57 in the normal type, each with a diameter of $4 \mathrm{~nm}$. The wavelength of the incident light is $\lambda=650 \mathrm{~nm}$ and the numerical aperture (NA) of the lens is 0.85 for TM polarization

coupled together and produce highly enhanced evanescent fields. Since the boundary conditions of Maxwell's equations stipulate that induced surface charge density is proportional to the discontinuity of the electric field component normal to the surface, only TM-polarized light can excite SPPs. These near-field optical signals of the Ag nanoparticles correspond to the nonlinear near-field optical properties previously found in experiments [18].

The Ag nanoparticles inside the bubble pits are assumed to be under the influence of a readout incident beam. The donut shape aperture (deformed $\mathrm{AgO}_{x}$ film) is formed only in the on-mark situation of the readout beam and provides the additional length (or path) longer than the normal length. In comparison with the evanescent fields for the normal type (Fig. 2b), the bubble pits of the deformed type (Fig. 2a) give additional outer boundaries to the motion of the $\mathrm{Ag}$ nanoparticles, and excite more evanescent fields, which are located at the far edge (top surface) of the bubble from the optical axis of the incident beam. It can be clearly seen in Fig. 2a that more evanescent fields extend in the direction of the $y$-axis (propagation direction). This indicates that

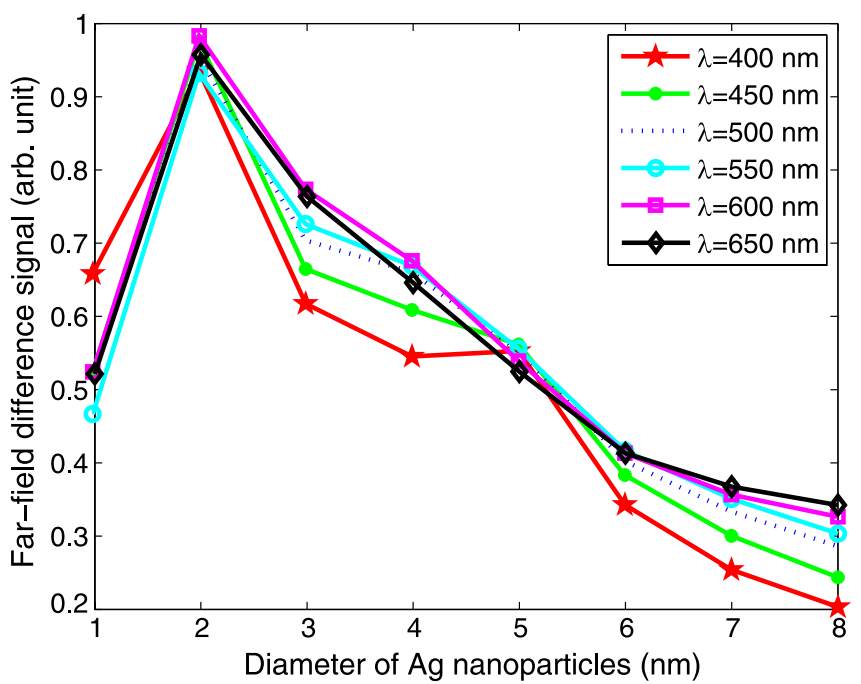

FIGURE 3 Calculated results of field intensity as a function of the diameter of Ag nanoparticles from 1 to $8 \mathrm{~nm}$, in increments of $1 \mathrm{~nm}$, measured at the top surface of the deformed $\mathrm{AgO}_{x}$ film at various wavelengths of incident light. The wavelengths of the incident light are 400, 450, 500, 550, 600 and $650 \mathrm{~nm}$, respectively

the bubble and particles tend to increase the readout signal intensity.

The optical responses of Ag nanoparticles are complicated, however, one key method for understanding these effects is to distinguish the contributions from each nanoparticle and particle-particle interactions as well as the gap between two nanoparticles. Surface plasmon polaritons and the scattering efficiency of Ag nanoparticles are usually sensitive to and controllable by particle size, the surrounding medium and the wavelength of the incident light. From experimental observation, the exact shape of an Ag nanoparticle is between a circle and a square. When the diameter of a nanoparticle is increased from $3 \mathrm{~nm}$ to $7 \mathrm{~nm}$, the shape becomes closer to a circle. With the same distribution of $\mathrm{Ag}$ nanoparticles as shown in Fig. 1, the field intensity measured at the surface of the deformed $\mathrm{AgO}_{x}$ layer varied considerably when they were illuminated by incident light at various frequencies (wavelengths). To verify the effect of the Ag nanoparticle size, the variation in near-field intensity measured at the top surface of the deformed $\mathrm{AgO}_{x}$ film with increasing diameter is tested. Figure 3 shows the field intensity as a function of the diameter of $\mathrm{Ag}$ nanoparticles from $1 \mathrm{~nm}$ to $8 \mathrm{~nm}$, in increments of $1 \mathrm{~nm}$, measured at the top surface of the deformed $\mathrm{AgO}_{x}$ film at various wavelengths of incident light. As the $n$ and $k$ values at different wavelengths of $\mathrm{Ag}$ and $\mathrm{AgO}_{x}$ can be found in [19-21], and corrected by the Drude model, which includes the size effect [22], we chose the wavelengths of the incident light to be 400, 450, 500, 550, 600 and $650 \mathrm{~nm}$ as examples, and the surface plasmon effects of other wavelengths can be verified by the same procedures. In the finite area, the height of the deformed $\mathrm{AgO}_{x}$ layer shown in Fig. 1 is $15 \mathrm{~nm}$. A smaller size of Ag nanoparticles means a lower density because the distance between two particles is larger. In the same manner, a larger size means a higher density cases because the gap between two particles is smaller.

The results in Fig. 3 clearly show that the size variations of Ag nanoparticles may yield different field intensities and 
indicate that the intensities of local fields exhibit a nonlinear optical response with increasing nanoparticle diameter. When the particle diameter is less than $1 \mathrm{~nm}$ (the lower density cases), shorter wavelengths (e.g., $\lambda=400 \mathrm{~nm}$ ) can excite more evanescent fields from the surface of $\mathrm{Ag}$ nanoparticles than longer wavelengths. When the particle size is larger than $1 \mathrm{~nm}$ (the higher density cases), the gaps between $\mathrm{Ag}$ nanoparticles are smaller and more local fields from adjacent nanoparticles are coupled together to produce highly enhanced evanescent fields. It can be seen in Fig. 3 that the field intensity at a fixed particle diameter of $2 \mathrm{~nm}$ exhibited peak values at the longer wavelengths, and these were the points where surface plasmon resonance occurred. This indicates that, at these wavelengths, the particle diameter of $2 \mathrm{~nm}$ provides a major contribution to plasmon coupling with the dipole radiation between the particles being the evanescent field of near-field light. Since the field propagation is predominantly taken as the near-field light, super-resolution exceeding the diffraction limit is made similar to that of nearfield scanning optical microscopy. When the particle size is larger than $2 \mathrm{~nm}$, the Ag nanoparticles form aggregative silver clusters which act as a silver bulk. The intensity decreases owing to absorption in this metal bulk and the destructive interference of SPPs in particle-to-particle interactions.

In order to compare the simulation results to the measured carrier-to-noise ratio (CNR) in an optical disk tester, the different of far-field intensity between the on-mark and the off-mark situations of the deformed super-RENS disks were computed for the various mark sizes. Figure 4 shows the differences of far-field intensity for the Ag nanoparticles of random distribution as shown in Fig. 1, with different wavelengths at a particle diameter of $2 \mathrm{~nm}$. These results were normalized against the far-field signals of the $\mathrm{Ge}_{2} \mathrm{Sb}_{2} \mathrm{Te}_{5}$ recording layer without recording marks, and varied considerably when they were illuminated by incident light at various frequencies (wavelengths).

In the observation of our simulations, if there was no $\mathrm{Ag}$ nanoparticle in the deformed active layer, the far-field signals dropped rapidly and became indistinguishable when the size of the recording marks were smaller than the optical diffraction limit $\lambda /(4 \mathrm{NA})$. At shorter wavelengths, the minimum distinguishable size of recording marks was smaller. When there were $\mathrm{Ag}$ nanoparticles distributed randomly in the deformed active layer, the far-field difference signals were several magnitudes higher and the recording marks were as small as $50 \mathrm{~nm}$ and were still distinguishable, i.e., recording marks smaller than $\lambda / 10$ could be read. Similar results have been obtained from the normal type (see Fig. $2 b$ ) from our previous results [6]. It can be observed in Fig. 4 that the farfield difference signals did not decrease monotonously with decreasing recording mark size or increasing incident wavelength. The far-field difference signals with smaller incident wavelengths (i.e., $\lambda=400,450,500 \mathrm{~nm}$ ) had a higher intensity than those of higher incident wavelengths (i.e., $\lambda=550$, $600,650 \mathrm{~nm}$ ) when the mark sizes were less than $100 \mathrm{~nm}$. The random distribution of embedded $\mathrm{Ag}$ nanoparticles in the deformed active layer influenced the near-field enhancements as well as the far-field signals, so the far-field signals had some complex variations, especially for the recording marks smaller than the diffraction limit. A distinct nonlin-

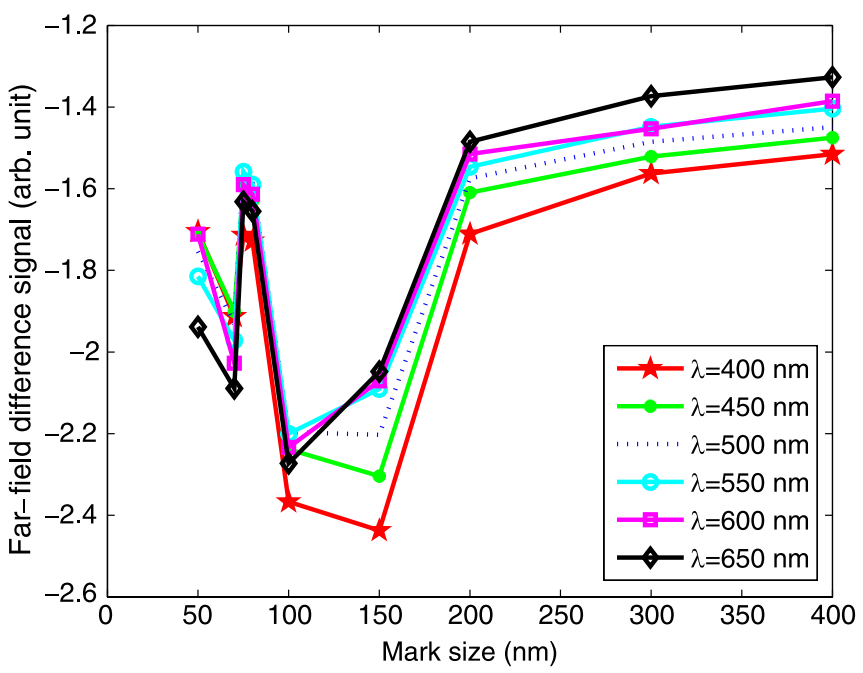

FIGURE 4 Far-field difference signals of the deformed $\mathrm{AgO}_{x}$-type superRENS disk with Ag nanoparticles. The wavelengths of the incident light are $400,450,500,550,600$ and $650 \mathrm{~nm}$, respectively

ear response of the deformed $\mathrm{AgO}_{x}$ thin film was shown in the results of our simulation, which agreed with our previous near-field and far-field measurements.

In further research, we will consider the model where not just a single bubble pit but two or more pits are in one spot. However, owing to the varying power of the incident light, the distribution and density of the nanoparticles may not be uniformly random in the area of the realistically deformed $\mathrm{AgO}_{x}$ layer. Further research into the effects of different distribution patterns may yield a better understanding of the optical properties of the deformed $\mathrm{AgO}_{x}$-type Super-RENS.

\section{$4 \quad$ Conclusion}

In conclusion, the deformation and plasmon effects of collective localized surface plasmons in a deformed $\mathrm{AgO}_{x^{-}}$ type super-RENS structure have been studied using the FDTD method. We found that the polarization direction, the wavelength of the incident light, and the size of the Ag nanoparticles are sensitive to the plasma resonance. The Ag nanoparticles inside the deformed $\mathrm{AgO}_{x}$-type super-RENS structure provide an additional length (or path) that is longer than the normal length (underformed case), and give additional outer boundaries to the motion of the Ag nanoparticles, and excite more evanescent fields that are located in the far edge of the bubble pit from the optical axis of the incident beam. The far-field signals of different wavelengths of incident light confirm the relation between highly localized near-field distributions and enhanced resolution of far-field signals. Our results can help us to design a super-RENS with superior resolution, as well as an alternative approach to near-field optical design for plasmon device, biological sensors and nanophotonic devices.

ACKNOWLEDGEMENTS The authors are thankful for the financial support from the National Science Council, Taiwan, R.O.C., under grant number NSC 95-2112-M-231-001, NSC-95-2119-M-009-029 and the Ministry of Economic Affairs, Taiwan, R.O.C., under grant number 95-EC17-A-08-S1-0006. 


\section{REFERENCES}

1 W.C. Liu, C.Y. Wen, K.H. Chen, W.C. Lin, D.P. Tsai, Appl. Phys. Lett. 78, 685 (2001)

2 H. Fuji, J. Tominaga, L. Men, T. Nakano, H. Katayama, N. Atado, Japan. J. Appl. Phys. 39, 980 (2000)

3 J. Tominaga, T. Nakano, N. Atoda, Appl. Phys. Lett. 73, 2078 (1998)

4 T. Fukaya, J. Tominaga, T. Nakano, N. Atoda, Appl. Phys. Lett. 75, 3114 (1999)

5 W.C. Lin, T.S. Kao, H.H. Chang, Y.H. Lin, Y.H. Fu, D.P. Tsai, C.Y. Wen, K.H. Chen, Japan. J. Appl. Phys. 42, 1029 (2003)

6 T.C. Chu, W.C. Liu, D.P. Tsai, Opt. Commun. 246, 561 (2005)

7 M.Y. Ng, W.C. Liu, Opt. Express 14, 9422 (2005)

8 T. Na, Y. Yamakawa, J. Tominaga, N. Atoda, Japan. J. Appl. Phys. 40, $1531(2001)$

9 F.H. Ho, W.Y. Lin, H.H. Chang, Y.H. Lin, W.C. Liu, D.P. Tsai, Japan. J. Appl. Phys. 40, 4101 (2001)

10 T. Kikukawa, A. Tachibana, H. Fuji, J. Tominaga, Japan. J. Appl. Phys. 42, $1038(2003)$
11 H. Fuji, T. Kikukawa, J. Tominaga, Japan. J. Appl. Phys. 43, 4212 (2004)

12 B.S. Lin, D.P. Tsai, W.C. Lin, ISOM Tech. Dig., Th-H-02 (2003)

13 J.B. Judkins, R.W. Ziolkowski, J. Opt. Soc. Am. A 12, 1974 (1995)

14 S.G. Stan, The CD-ROM Drive, A Brief System Description (Kluwer, Boston, 1998), Chapt. 2.3, p. 16

15 A. Taflove, Computational Electrodynamics (Artech House, BostonLondon, 1995), p. 203

16 J.B. Judkins, R.W. Ziolkowski, J. Opt. Soc. Am. A 12, 1974 (1995)

17 T.-C. Chu, W.-C. Liu, D.P. Tsai, Scanning 26, I-102 (2004)

18 T. Nakano, A. Sato, H. Fuji, J. Tominaga, N. Atoda, Appl. Phys. Lett. 75, 151 (1999)

19 C. Liu, J. Erdmann, J. Maj, A. Macrander, J. Vac. Sci. Technol. A 17, 2741 (1999)

20 T. Shima, J. Tominaga, Japan. J. Appl. Phys. 42, 3476 (2003)

21 P.B. Johnson, R.W. Christy, Phys. Rev. B 6, 4370 (1972)

22 T. Okamoto, in: Near-Field Optics and Surface Plasmon Polaritons, ed. by S. Kawata (Springer, Berlin, 2001), p. 99 